\title{
An Analysis of Factors Influencing Transmission of Internet Memes of English-speaking Origin in Chinese Online Communities
}

\author{
Siyue Yang \\ Shanxi Normal University, Linfen, China
}

\begin{abstract}
Meme, as defined in Dawkins' 1976 book 'The Selfish Gene', is "an idea, behaviour or style that spreads from person to person within a culture". Internet meme is an extension of meme, with the defining characteristic being its spread via Internet. While online communities of all cultures generate their own memes, owing to the colossal amount of content in English and the long \& widespread adoption of Internet across all strata of society in English-speaking countries, the vast majority of high-impact and well-documented memes have their origin in English-speaking communities. In addition to their spread in the original culture sphere, some of these prominent memes have also crossed the cultural boundaries and entered the parlance of Chinese Internet communities. This paper seeks to give a brief introduction to Internet memes in general, and explore the factors that control and/or facilitate a meme's ability to enter Chinese communities.
\end{abstract}

Index Terms - Internet meme, cross-cultural communication, Chinese Internet

\section{INTRODUCTION}

Internet meme, an extension of the term "meme" first coined by Richard Dawkins (1976) in his work The Selfish Gene (p. 192), refers to the unique form of meme that spreads through the Internet. Internet memes in their various forms currently enjoy substantial popularity among Internet users all around the globe, and are flourishing and becoming increasingly entrenched in the mainstream culture of all the disparate societies in this connected world. Scraps of information online are gaining and losing their meme status by every passing day, through large-scale replication and selection by the legions of interested Internet users.

An example of Internet meme that is fairly close to the daily life of almost all of Chinese Internet users would be the various images used by users of instant messaging services such as Tencent QQ as canned expressions, commonly referred to as "emotes" or "stickers". While normally not thought as such, the stickers do indeed express all the properties as units of cultural transmission, and undergo processes of replication, transmission, mutation and selection when used by the users of instant messaging services. Replication and transmission occur when the images are sent to other users and appropriated by others for their own use, the modification of the images and creation of derivatives corresponds to mutation, and selection is the basis of the rise and fall of the frequency of appearance of different images in chats.

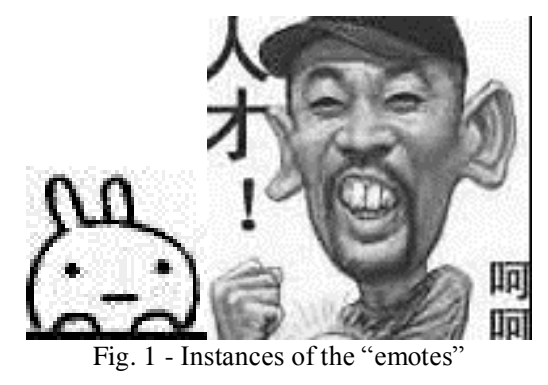

While every community online generates its own memes, owing to the colossal size and maturity of English-speaking Internet, the vast majority of Internet memes that achieved worldwide success have their origins in English-speaking communities, and among these memes are some outliers which have also managed to penetrate the Chinese Internet. What are these memes? How did they enter Chinese online communities, despite the great differences in culture and language? This paper seeks to answer these questions.

\section{Meme AND InTERnEt Meme}

An explanation of the concept of meme and Internet meme is necessary before we are to move on and discuss the memes themselves. As is mentioned in the introduction, the concept of meme comes from Dawkins' 1976 work, 
invented to describe the humanity's cultural evolution, as the basic unit of information existing in brain and the mental space, and as an analogue to the gene - the vital part of biological evolution, sharing various characteristics with it, especially its nature as replicator. Some of the examples of common memes are tunes, ideas, catchphrases, and so on. (p. 192)

Internet meme, obviously, refers to a meme that is propagated over the globally-connected network, the Internet. Yet the simplistic explanation proved to be insufficient, as it is found that the concept of Internet meme carries certain characteristics that differentiate itself from the traditional concept of meme - the memetics researcher, Susan Blackmore even went so far as defining Internet meme as belonging to a new subset to the concept of meme called temes- a wholly new category of replicators, memes which live in technological artifacts instead of human minds (Zetter, 2008). It is observed that the typical contemporary Internet meme propagates among people by means of email, instant messaging, forums, blogs or social networking sites, and commonly consists of offbeat news, websites, images or video clips (Bauckhage, 2011). Also of note is that unlike traditional memes that reside in traditional media, which render tracing and analysis of the propagation of a particular meme difficult, the utilization of Internet as the medium by Internet memes lends to comparatively easier retrieval and analysis of the track of transmission: digital tracks are left behind whenever an email is sent, a message is posted on a bulletin board, or a chat is initiated over an instant messaging platform, and the longevity and fidelity of data on digital storage devices (Blackmore 2000, p. 216) plus the existence of powerful search engines both greatly facilitates the study of Internet memes - which this study took particular advantage of.

\section{RELATED AND PRIOR WORK}

The study of memetics has a relatively late start in Chinese academia, with initial attention only brought to the field as late as 2002 with a mention of the concept of meme and a suggestion for further research in a preface for the book Language and the Culture $(\mathrm{Gu}, 2002)$. Following $\mathrm{Gu}$, one of the more prominent early researchers in the field of memetics would be He Ziran, whose works (He, 2003; 2005) served to introduce the theory to a broader audience, and to pioneer in employing the theories of memetics in the study of language. Pertaining to the current study, it is unfortunate that despite the popularity of the research on the Internet memes in the Western academia (see below), studies at home that deal directly with the phenomenon of Internet memes by itself, unbound to subjects such as online language or computer-meditated language use, remained scarce. Nevertheless, this paper draws inspiration from early studies on the use of online language/"netspeak" by Li (2006), Cao (2008), and Tan (2011). Of particular note is the work by $\mathrm{Li}$, which proposed a set of factors affecting the success of online language and incorporated the same framework in justifying the research as in this paper, and served as an important reference for this paper.

For western studies, aside from the works by Dawkins (1976) and Blackmore (2000) which were introduced in the section prior to this one and which serve as the cornerstones of the study of the memetics, this study specifically owes its theoretical framework upon which the entire analysis relies on to Heylighen's influential works (1996; 1999) on the replication of Internet memes on the Internet, and the selection criteria of meme replication. One interesting line of development in study of memes in the western academia is the empirical studies that go beyond the theoretical, qualitative inquiries into memes, and which sought to establish and test predictive models of meme propagation: such as Bauckhage (2011); Adar and Adamic (2005); Lin et al. (2010). While not overly relevant to the current study, these studies have provided certain valuable insights into the spread of Internet memes, and the use of research tools such as online meme databases and search engine statistics were incorporated into this study.

\section{THE PRESENT STUDY}

This study seeks to understand what are potential factors affecting the success of a particular meme in transgressing the cultural boundaries; specifically, what factors made certain memes which have their origin in the English-speaking Internet, a completely different cultural context, succeed in entering the Chinese online communities and gaining popularity among Chinese Internet users; and how these factors fit within the meme replication \& selection framework postulated by Heylighen (1999). Therefore, the research questions are as follows:

1. What are the factors leading to the success of certain Internet memes of English origin in achieving cross-cultural transmission and entering Chinese online communities?

2. How do these factors relate to the meme replication selection criteria model?

These questions will be answered through comparative analysis of several examples of extant memes originating in the English-speaking Internet, of which some achieved mainstream popularity among Chinese Internet users in relatively intact form, and the rest failed in managing to achieve cross-cultural transmission despite having relatively similar forms and a comparable level of popularity in the original cultural context. The following section details the memes used in this study.

\section{MEMES IN ThIS STUDY}

\section{A. Examples of Successful (Achieved Prominence in Chinese-speaking Online Communities) Memes}




\section{Downfall / Hitler Reacts}

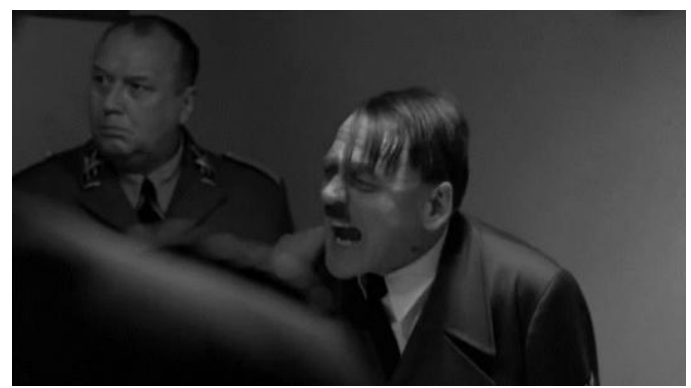

Fig. 2 - One of the iconic scenes in Downfall, which is essentially always present in parodies.

The "Downfall" is a series of parody-subtitled videos of a scene from the 2004 German film Der Untergang, a World War 2 epic drama. The scene depicts Hitler, upon learning from the top military commanders that the defeat of Germany is inevitable, orders everyone to leave except the four highest ranking officials, succumbs to a breakdown, and starts a rant full of scathing attacks on the upper echelon of Nazi military before calming down and resigning to fate.

Content creators quickly made use of the language barrier and started to create parody videos from various segments from the film. Taking advantage of the particular scene's original acting and audibly raging voice of Hitler, videos with parody, anachronistic subtitles in English that were angry rants generally expressing disbelief and anger at what the authors were against, or had strong opinion on quickly popped up ("Downfall / Hitler Reacts", n.d.). Many similar videos followed, typically discussing current events and trivial news. The phenomenon was quickly covered by Wired (Wortham, 2008) and Laughing Squid (Beale, 2008), and later by New York Times Magazine (Heffernan, 2008).

While it cannot be reliably determined which video is the earliest instance of Downfall parodies subtitled in Chinese, searching suggests that the earliest one is dated November 12, 2008, and is a direct translation of a parody originally in English. This video is relatively unremarkable - Hitler, upon learning that his gaming console was banned from the multiplayer platform, goes on a rant against Microsoft. Needless to say, the appeal of the video was rather limited and the content prevented the parody from gaining influence outside of gaming circles.

The Chinese derivations of the Downfall meme saw a significant surge in mainstream popularity in 2010, during the height of the 360 v. Tencent disputes over competitive practices. A parody, titled "Exposed: Tencent's Confidential Anti-360 Meeting" (2011) was uploaded to Youku, the leading Chinese video website, and received considerable attention from Chinese Internet users, totaling upwards of 2 million playbacks (as recorded by Youku). The meme was then commonly referred as "Fury of the Führer" on Chinese Internet.

2. Doge

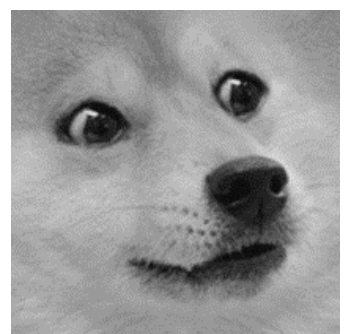

Fig. 3 - One of the more iconic pictures of the "doge"

"Doge", an intentional misspelling of "dog", is a meme (in its initial form) associated with a picture of a specific Shiba Inu (or in its earlier form and some of the current instances, any Shiba Inu) accompanied by multicolored text in Comic Sans in the foreground. The text represents a form of internal monologue, and is almost always written in deliberately broken English with an overabundance of the five modifiers "so", "such", "much", "very" and "many", usually appearing at the beginning of every line. The modifiers are applied in such a way that the noun phrase is always ungrammatical.

The particular picture associated with the Doge meme is a Shiba Inu named Kabosu, rescue-adopted by a Japanese kindergarten teacher Atsuko Sato. This peculiar picture of Kabosu, sitting on a couch and glancing sideways at the camera with raised eyebrows, was uploaded along with other photos by Ms. Sato to her personal blog. Later in the year, a photo of Kabosu was submitted to the /r/Ads section on the popular news aggregation site reddit, and received some attention from its users. This marked the first known and well-documented posting of the picture on English-speaking Internet ("Doge", n.d.). It took some time for the meme to evolve to its current form - initially merely included as one photo in a variety of other pictures of dogs used as backgrounds of internal monologues, and in a set of photos of Shiba Inus used as backgrounds for a single-topic tumblr blog "Shiba Confessions", the Doge later garnered enough popularity - probably thanks to the peculiar pose of the picture and its general cuteness - to become one single Internet meme by itself, and the styling of the internal monologue put on Doge pictures is actually derived from the style used 
on the "Shiba Confessions" blog. Besides than the internal monologue form, another form of the Doge meme consists of digitally altered images of a Shiba Inu - often Kabosu, or the face of Kabosu placed in other pictures.

The spread of Doge meme on Chinese Internet saw relatively little use of the Internet meme in its original form - the internal monologue, conveying a humorous overtone through intentional misspelling and ungrammatical wording, did not resonate well with a Chinese audience due to the presence of the language barrier. Instead, many Chinese netizens became "enchanted" by the peculiar picture of Kabosu and the meme proliferated on Chinese Internet in its other form, in the digitally altered photos and derivational artworks of the Shiba Inu.

Casual observation alone is enough to reveal Doge's widespread use on Chinese Internet. While the popularity might have waned these days, it was almost impossible to avoid seeing a Doge image or two in discussion groups with a reasonable number of young members, of whatever instant messaging tool one uses. Searching the keyword "doge" with the Baidu search engine now (as of May, 2017) yields tens of millions of results, ranging from the original image, simple altered images to original derivational artworks and even more amusing, picture of a certain actress that some pointed out looked like the Doge. Another powerful evidence of Doge's influence would be Sina Weibo's tweet emoticon list, which at the moment prominently features a simplistic emoticon derived from Doge in the first row of the first page, aptly named [doge].

3. Rage Comics

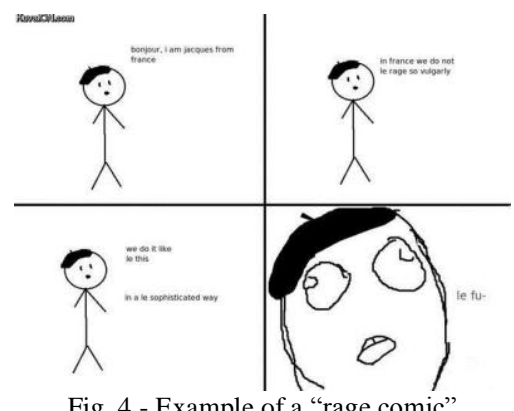

Fig. 4 - Example of a "rage comic"

"Rage Comics" refers to series of webcomics featuring characters sometimes called "rage faces" that are simplistic line drawings made in entry level drawing software such as Microsoft Paint. The theme of the comics is not strictly limited to any particular genre, but normally the stories reflect real-life experiences of the authors. As implied by the name of the Internet meme, Rage Comics often tell stories of authors' grievances and other negative experiences in life and express anger and/or frustration on certain subjects.

The pool of characters available in Rage Comics, the "rage faces", started relatively small but is steadily growing, with newer additions enjoying varying levels of popularity. Some of the common early characters include the following: Rageguy, Trollface, Okay Guy, Forever Alone, which represents rage, trolling (intentionally expressing controversial/incorrect opinions for dramatic effect), resignation in face of the realities of daily life ("everything is okay"), and inability to find a partner due to personal defects and peculiarities.

Aside from the characters, there are also several terms and styles unique to Rage Comics, but usage of these are not always required or necessary. For example, "le", a French article, is often placed at the beginning of a sentence to signify action or used as a general replacement of "the". This custom originated from a comic from the early days of Rage Comics, which unsurprisingly was about French people and used "le"s liberally. In another example, two words, "herp" and "derp" are often used as words indicating stupidity; they are used as placeholders for unimportant dialogues, replacement word for stupid/unimportant actions, or as names for characters in a comic, often in a slightly altered form (e.g. "Derp Herpington", "Derpth of Herpuary".) ("Rage Comics”, n.d.).

Rage Comics entered Chinese Internet communities in 2008, and saw rapid dissemination through social networking websites. The meme went through considerable localization on Chinese Internet - unique Chinese names were created for the characters and creators, mostly free from knowledge of the dogmas and formulas laid down by the Rage Comics' early creators, developed their own styles and used the characters liberally - few retained their original meaning in Chinese versions of Rage Comics. In 2010, the addition of the "Yao Ming Face" character to the pool of Rage Comics characters greatly boosted its popularity. Possibly motivated by this addition, new characters featuring faces of Chinese celebrities, that are either unique to Chinese Internet or shared with the global community of Rage Comics creators soon emerged - examples include Jackie Chen, Fengjie, the poet Du Fu, and so on.

In 2012, the spread of Rage Comics meme on Chinese Internet was further boosted by the creation of a website dedicated to creation and hosting of Chinese Rage Comics ("Rage Comics - An Internet Cultural Brand Belonging to the Young", 2015). Consider that the entry barrier of creation of new instances of the Rage Comics was already low enough - presence of pre-made, canned characters meant that creation required little to no artistic skills, only a story in head plus most basic skills in using drawing software to copy-paste characters and adding subtitles. The launching of the website made Rage Comics creation even more accessible to average Internet users by streamlining the creation process and handling the hosting of images and comments. 
The meme also went beyond humor and was picked up by commercial interests - The "Rage Big Events" series, which is a popular online talk show launched in 2013 by the company behind the aforementioned website, features a host wearing masks of characters in Rage Comics (ibid.). In 2012, a crudely drawn advertisement featuring Rage Comics character for an online store went viral on Chinese Internet, and thanks to the humor and easily recognized characters the store reportedly experienced a spike in sales during the lifetime of the advertisement ("Rages Comics Becoming Advertisement", 2012). Slogans originating from Rage Comics - for example "destined to be alone forever", a derivation of "forever alone" - have also entered common parlance of Chinese Internet users. All in all, over years the Rage Comics meme has already integrated itself into the online culture of Chinese Internet.

\section{B. Examples of Memes of Comparable Popularity Restricted to English-speaking Internet}

\section{LOLcats}

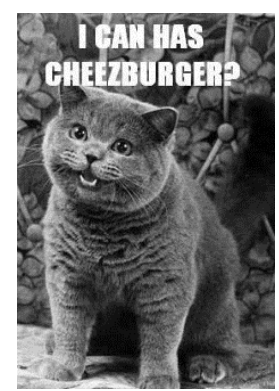

Fig. 5 - An example of LOLcats, with the stock phrase "I can has cheezburger?"

LOLcats is an Internet meme with a main format consisting of a randomly picked cat picture (that is considered to be cute or otherwise attractive) plus captions in a specific style, "lolspeak". The style is intended to convey a feeling of immaturity and cuteness through the use of ungrammatical constructions and intentional misspelling - for example, the common stock phrase in LOLcats memes, "I can haz cheeseburger?" (Silverman, 2007; "Lolcat", 2017). The style is not as freeform as the description might suggest - certain established forms are present, such as the tendency to write "s" as "z" and the common recurring phrasal form "Im in ur [noun of item] [verb of activity related to item]-ing ur [noun related to first item]." LOLcats' popularity can be seen through the coverage it has received on the mainstream media. On June $12^{\text {th }}, 2007$, Time Magazine (Grossman, 2007) reported that a Google search for "lolcat" then yielded over 3.3 million hits; the meme went so far as sparking academic interest in research on Internet memes and the LOLcats itself, which is covered in an article from the Atlantic (Rosen, 2012) in May, 2012.

2. All Your Base Are Belong to Us

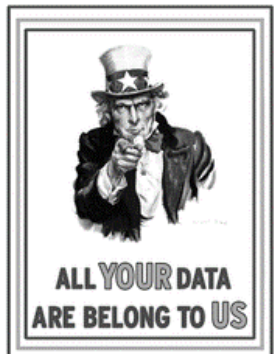

Fig. 6 - A variation on the phrase in a poster protesting mass surveillance of the US government

"All your base are belong to us" is a popular, albeit somewhat historical early Internet meme based on the catchphrase that has its origin in a broken, mistranslated English phrase found in the opening cutscene of the 1992 Mega Drive video game Zero Wing ("All your base are belong to us", 2017). The meme was popularized by an animation of the opening text of the game which gained considerable popularity on the Something Awful forums (Dibbel, 2008), and later on the phrase or variation thereof has appeared in countless articles, books, images, games, comics, and so on. One of the testaments to the influence of the phenomenon is the Wired (Benner, 2001) report on it, covering its propagation from its origin as animation to its then current status as a major online catchphrase that even managed to make strides in the real life in the form of T-shirts. Another case of the meme being used which demonstrated its popularity among Internet users was the appearance of the phrase on the front page when the world's largest video sharing website YouTube was taken down for maintenance in 2006 (Sandoval, 2006).

3. Do a Barrel Roll 


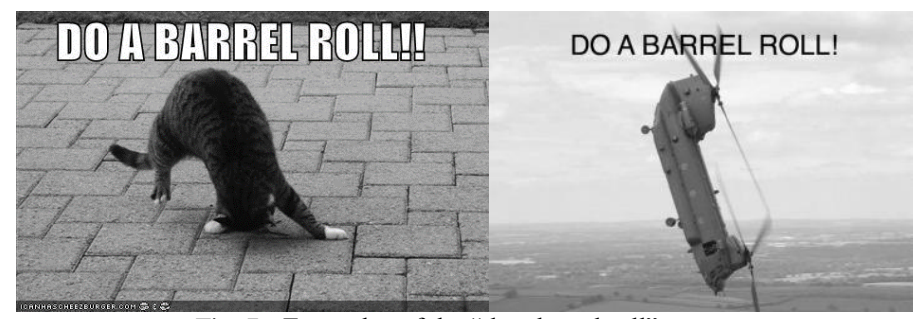

Fig. 7 - Examples of the "do a barrel roll” meme

"Do A Barrel Roll" is a catchphrase used to call on someone to perform a 360 degree horizontal spin, and comes from the 1997 video game Star Fox 64 where one of the characters in the game orders the player to do a barrel roll. The phrase is often used to caption images where the subject appears to be in mid-rotation, or in case of animations where the subject is performing a full rotation, as seen in the examples ("Do A Barrel Roll”, n.d.). One of the meme's earliest recorded spike of popularity on the imageboard 4chan dates back to August $2^{\text {nd }}, 2007$, and the meme has managed to achieve such recognition that the world's largest search engine provider, Google, actually implemented an easter egg function for the search keywords "do a barrel roll" which upon entry would rotate the screen for 360 degrees clockwise. (Burns, 2011)

\section{COMmOn Properties Influencing SuCCESSFul Cross-CUltural Transmission OF MeMES}

Analyzing the examples of successful and unsuccessful (judged by whether the specified Internet meme of Englishspeaking communities origin achieved a high popularity in Chinese online communities) Internet memes, three common properties influencing the successful transmission of an Internet meme across cultural boundaries can be isolated; specifically, 1) the significance of the verbal component, 2) the ability to evoke feelings without relying on the original cultural background, either universally or in a way adapted to the new culture, and 3) the presence of external actors promoting the transmission of a certain meme for any purpose.

The first two properties are present in the successful examples of Internet memes and noticeably absent in the unsuccessful examples - which are by themselves memes of considerable popularity and success, thus removing any possibility that the memes were merely incapable of standing on their own at all - pointing to their relative importance in cross-cultural transmission of Internet memes. The last property, presence of external actors promoting the meme, is present in a significant manner in one of the examples and generally present for other examples, but due to its nature generally cannot be found in unsuccessful examples. Thus, the author considers it to be a minor factor and only includes it for the sake of completeness.

1. Significance of verbal component in original language

The first factor, significance of verbal component refers to the position text and other verbal information occupies in a particular meme. Memes which rely heavily on the verbal component to express meaning are considered to be dependent on the verbal component, and vice versa. Also of note is that the term "verbal component" here refers to verbal content in the original language of the Internet meme that must be retained, or transformed to a form closely related to and conveying precisely the same meaning as the original form.

Each of the examples of successful memes exhibits a low dependence on unchanged verbal content, while none of the unsuccessful cases does. In case of Hitler Reacts and Rage Comics memes, the verbal component can be replaced entirely without any detrimental effect to the transmission of the memes; and in case of Doge, the verbal component is often simply discarded. On the other hand, LOLcats depends heavily on the presence of intentionally ungrammatical and misspelling-ridden language to express its meaning, and the pictures of cute cats by themselves lack a distinct character to be the sole carrier for the meaning of the meme. The same goes for the All Your Base and Do a Barrel Roll memes as well, both placing significant importance on the verbal component due to their basic form as catchphrases and derivations of so.

2. Able to evoke feelings without reliance on the original culture

Survival and further transmission of an Internet meme in communities which do not share a common cultural background as its origin obviously depends on its ability to evoke feelings and emotions, without requiring a cultural background that the new, prospective host might not necessarily have.

The successful examples mentioned before in this paper are all capable of evoking feelings in a way that is independent of their original cultural background, but through slightly different means. All of the successful memes achieve universal appeal through pandering to universal human feelings, with information that is universally recognized and beyond any specific cultural setting. It's easy to understand Doge's appeal: by exploiting people's curiosity and fondness of cute animals, with graphic information that is well understood in different cultures (note that the picture originated in Japan, and only later gained unprecedented popularity on English-speaking Internet), the meme managed to conquer many without even needing accompanying text or a shared cultural background, as recognition of cute animals is universal (Golle et al. 2013).

The Downfall and Rage Comics, however, also take a different approach in addition to being universal. All three have a significant graphic component, and are easily processed by new hosts. But it should also be noted that they all 
often contain a host-generated verbal component too, which is adapted to the cultural setting they're currently in. This component, as stated before in the descriptions of the respective memes, generally expresses the frustrations and complaints of the author, documents frustrating events in daily life, or presents self-deprecating humor. All of these are rather easy for other members of the same cultural setting to understand and sympathize with.

For a negative example, consider the Barrel Roll meme. Although superficially the meme can be easily understood by anyone, its real power, the factor that powered its spread is its origin as a part of a shared cultural heritage. Being a derivation of a notable scene in a best-selling video game, the meme is well capable of reminding the majority of those who make and spread memes - young adults their childhood memories, and thus evoking a feeling of familiarity. What made it successful in English-speaking communities is likely also the reason of its failure elsewhere as the meme is too tightly integrated into one culture and one shared memory, that it becomes incomprehensible for those who don't share the same heritage - such as the majority of Chinese netizens.

3. Presence of external actors promoting the transmission

Certain memes of foreign origin that managed to enter Chinese online communities were also helped in popularity by external actors, who have more social influence than any average netizen or host of an Internet meme, intentionally pushing for their transmission for various reasons. A cursory survey of the Sina Weibo microblogging platform revealed that the examples of successful memes introduced before were/have been used for promotion of marketing accounts, to draw attention to other content provided. While these actions are motivated entirely by selfish reasons, it cannot be denied that they in fact helped to promote the memes in question at the same time, and acted as powerful external actors in the meme lifecycle.

Besides memes merely being used for promotion, an unusual case is the Rage Comics meme which, as is described before in this paper, actually received significant investment from an external actor by itself. However, the final result, the boosting effect on the meme's popularity, remains the same.

\section{Analyzing the EfFect of the Properties Under the Meme RePlication Selection CRiteria Model}

Heylighen's (1999) model for a meme's replication lifecycle breaks the process of meme replication through hosts into four stages of assimilation, retention, expression and transmission, with different types of "selectors", traits of memes that determine the fitness for survival of a certain meme at each stage. Situating the three properties, expressed in the aforementioned Internet memes that managed to transgress the cultural boundaries, within the framework of the model allows us to explain how these properties benefit the memes in question in the competition for survival. Analysis in this interpretation suggests that the properties translates to benefits to survival and further transmission through their effects primarily on the selectors in the subjective and inter-subjective groups, specifically in simplicity, coherence, utility, publicity and expressivity. The item-by-item breakdown is as follows.

1. Simplicity

Defined as "requiring less processing for the meme to be understood", it is readily apparent that both low dependence of verbal content and of shared cultural background serve to lower the difficulty in assimilating the meme for a host who neither speaks the language of the original meme, nor shares a common cultural background; as is the case in the transmission of a meme originating from English-speaking communities to the Chinese Internet.

\section{Coherence}

The coherence selector refers to the connection and consistency between old memory traces and new perception, or in other words the ability for preexisting concepts to support the newly incorporated information. The independence from specific cultural backgrounds as displayed by the examples of "successful" memes in general allows the memes to appear more coherent, or appeal to baser concepts such as emotion or universal recognition of facial expressions, etc. and allow themselves to be supported by these preexisting concepts, instead of a presumably shared cultural background which may or may not exist in all situations - a defect especially obvious in cases of cross-cultural transmission.

3. Utility and expressivity

For an Internet meme, of which almost the only purpose to its hosts is to be shared to the others for benefits in either an interpersonal (social relationship, popularity in social group) or material (in case of Internet personalities, actual return for "clicks") sense, the utility of a meme is closely related to its expressivity; in other words, expressivity equals to utility for most of the Internet memes, and any improvement in expressivity ("the ease with which a meme can be expressed in an intersubjective medium") results directly in an increase in utility to the host. Similar to the case for the simplicity selector, low dependence on verbal content in a specific language and low dependence on a shared cultural background helps in lowering the difficulty for a Chinese-speaking host to express a meme with origin in Englishspeaking communities by allowing the textual content to be eliminated or changed to a form more acceptable, and posing no difficulty in interpreting a unfamiliar cultural background. The expressivity advantage, in turn, translates directly to an advantage in utility to the hosts.

4. Publicity

Heylighen defined the publicity selector as the efforts by host(s) put into broad distribution of the message. The effect of having an external actor with significant influence actively seeking to promote an Internet meme of English origin mostly manifests within the domain of publicity, where the host devotes higher amount of resources to 
transmission of a meme for own purposes (see before for utility of an Internet meme in terms of Internet personalities), thus adding into a meme's publicity and promoting its fitness for survival.

\section{CONCLUSIONS}

In this paper, several prolific Internet memes of English-speaking communities origin that achieved exceptional popularity were investigated, their common properties which were crucial in gaining acceptance in the completely different linguistic and cultural settings that is the Chinese online communities summarized. Analyzed in comparison with the aforementioned memes were Internet memes of considerable popularity in English-speaking communities that failed to gain recognition in China, which appeared to lack the properties displayed by the more successful Internet memes. The common properties were placed in and analyzed with the meme lifecycle model proposed by Heylighen (1999), and it is concluded that the properties benefit the memes in fitness for survival in all four stages of meme transmission, through the selectors in subjective and inter-subjective group - specifically, simplicity, coherence, utility, expressivity and publicity.

It is apparent that Internet memes will remain to be an integral part of the online culture for the foreseeable future. Countless Internet memes are being born, transmitted, replicated, discarded and otherwise used in all kinds of ways in online communities all across the globe at this very moment, and some of these would manage to gain a popularity significant enough to enter the mainstream parlance, cross cultural boundaries and eventually become a common part of culture shared by millions, or even billions. The importance of studying a phenomenon that is affecting essentially everyone in this online age cannot be dismissed; the nature of Internet memes' primary media also means that often very visible and traceable digital footprints are left behind occurrences of transmission and replication, which makes the phenomenon extra conducive to future research and studies. The author hopes that this paper would spur some academic interest in this field of inquiry and lead to further research on the subject of cross-cultural transmission of Internet memes.

\section{REFERENCES}

[1] All your base are belong to us. (2017). In Wikipedia. Retrieved May 14, 2017, from https://en.wikipedia.org/w/index.php?title=All_your_base_are_belong_to_us\&oldid=775191407.

[2] Bauckhage, C. (2011). Insights into Internet Memes. In Proceedings of the Fifth International AAAI Conference on Weblogs and Social Media.

[3] Beale, S. (2008). Hitler Downfall Mashup Video Meme Über Alles. Retrieved May 15, 2017, from https://laughingsquid.com/hitler-downfall-mashup-video-meme-uber-alles/.

[4] Benner, J. (2001). When Gamer Humor Attacks. WIRED. Retrieved May 15, 2017, from https://www.wired.com/2001/02/when-gamer-humor-attacks/.

[5] Blackmore, S. (2000). The Meme Machine. Oxford, England: Oxford University Press.

[6] Burns, M. (2011). Do A Barrel Roll! In Google! Right Now! [Blog]. Retrieved May 14, 2017, from https://techcrunch.com/2011/11/03/do-a-barrel-roll-in-google-right-now/.

[7] Cao, J. (2008). Imitation and Copying in Transmission of Net Speech. In 2nd National Academic Conference of Doctoral Students in Journalism and Media Studies at Communications University of China (p. 8). Beijing: Communications University of China.

[8] Coscia, M. (2013). Competition and Success in the Meme Pool: A Case Study on Quickmeme.com. Presented at the International AAAI Conference on Web and Social Media. Retrieved May 11, 2017, from https://www.aaai.org/ocs/index.php/ICWSM/ICWSM13/paper/view/5990/6348.

[9] Dawkins, R. (1976). The Selfish Gene. Oxford, England: Oxford University Press.

[10] Dibbell, J. (2008). Mutilated Furries, Flying Phalluses: Put the Blame on Griefers, the Sociopaths of the Virtual World. WIRED. Retrieved May 14, 2017, from https://www.wired.com/2008/01/mf-goons/.

[11] Do A Barrel Roll. (n.d.). In Know Your Meme. Retrieved May 15, 2017, from http://knowyourmeme.com/memes/do-a-barrelroll.

[12] Doge. (n.d.). In Know Your Meme. Retrieved May 15, 2017, from http://knowyourmeme.com/memes/doge.

[13] Downfall / Hitler Reacts. (n.d.). In Know Your Meme. Retrieved May 15, 2017, from http://knowyourmeme.com/memes/downfall-hitler-reacts.

[14] Exposed: Tencent's Confidential Anti-360 Meeting. (2011). [Video file]. Retrieved May 14, 2017, from https://v.youku.com/v_show/id_XMjE4NDgzMTg0.html.

[15] Golle, J., Lisibach, S., Mast, F. W., \& Lobmaier, J. S. (2013). Sweet Puppies and Cute Babies: Perceptual Adaptation to Babyfacedness Transfers across Species. PLoS ONE, 8(3), e58248. https://doi.org/10.1371/journal.pone.0058248.

[16] Grossman, L. (2007). Creating a Cute Cat Frenzy. Time. Retrieved May 15, 2017, from https://content.time.com/time/magazine/article/0,9171,1642897,00.html.

[17] Gu, J. (2002). Language and Culture. (S. Lu, Ed.). Shanghai, China: Shanghai Foreign Language Education Press.

[18] Heffernan, V. (2008). The Hitler Meme. The New York Times Magazine. Retrieved May 14, 2017, from http://www.nytimes.com/2008/10/26/magazine/26wwln-medium-t.html.

[19] Heylighen, F. (1996). Evolution of Memes on the Network: from chain-letters to the global brain. In G. Stocker \& C. Schöpf (Eds.), Memesis: the future of evolution (pp. 48-57). Vienna: Springer.

[20] Heylighen, F. (1998). What Makes a Meme Successful? Selection Citeria for Cultural Evolution. In Proceedings of 15th International Congress on Cybernetics (pp. 413-418). Namur, Belgium: Association Internat. de Cybernétique. 
[21] Li, G. (2006). The Survival of Computer-Mediated Language - A Memetic Approach (Unpublished master's thesis). Guangdong University of Foreign Studies, Guangzhou, China.

[22] Lolcat. (2017). In Wikipedia. $\quad$ Retrieved $\quad$ May $15, \quad$ 2017, from https://en.wikipedia.org/w/index.php?title=Lolcat\&oldid=777415850.

[23] Rage Comics. (n.d.). In Know Your Meme. Retrieved May 16, 2017, from http://knowyourmeme.com/memes/subcultures/ragecomics.

[24] Rage Comics - An Internet Cultural Brand Belonging to the Young. (2015). Retrieved May 16, 2017, from http://www.xdz.com.cn/info/20718/147888.htm.

[25] Rage Comics Becoming Advertisement. (2012, September 29). Southeast Express, p. A14.

[26] Rosen, R. J. (2012). Are LOLCats Making Us Smart? The Atlantic. Retrieved May 16, 2017, from https://www.theatlantic.com/technology/archive/2012/05/are-lolcats-making-us-smart/256830/.

[27] Sandoval, G. (2006). YouTube: Our humor, not our hack. CNET. Retrieved May 14, 2017, from https://www.cnet.com/news/youtube-our-humor-not-our-hack/.

[28] Silverman, D. (2007). Web photo phenomenon centers on felines, poor spelling. Chron. Retrieved May 16, 2017, from http://www.chron.com/business/silverman/article/Web-photo-phenomenon-centers-on-felines-poor-1809345.php.

[29] Tan, X. (2011). The Study of Meme in Network Language Spreading. Journal of Jilin Normal University (Social Sciences), (03), 57-59.

[30] Wortham, J. (2008). Hitler Remixes Are Big - on YouTube. WIRED. Retrieved May 15, 2017, from https://www.wired.com/2008/05/adolf-hitler-is/.

[31] Zetter, K. (2008). Humans Are Just Machines for Propagating Memes. Retrieved May 15, 2017, from https://www.wired.com/2008/02/ted-blackmore/.

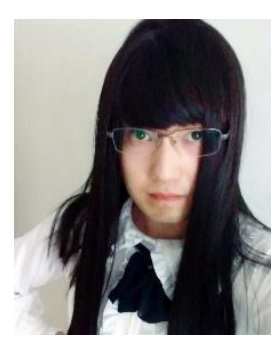

Siyue Yang was born in Taiyuan, China in 1992. He received his B.A. degree in English from Business College of Shanxi University, China in 2015.

$\mathrm{He}$ is currently a graduate student in linguistics in the School of Foreign Languages, Shanxi Normal University, Linfen, China. His research interests include sociolinguistics and language on the Internet. 